\title{
Common Coupled Fixed Point Theorems for Two Hybrid Pairs of Mappings under $\varphi-\psi$ Contraction
}

\author{
Bhavana Deshpande and Amrish Handa \\ Department of Mathematics, Govt. PG Arts \& Science College, Ratlam, Madhya Pradesh 457001, India \\ Correspondence should be addressed to Bhavana Deshpande; bhavnadeshpande@yahoo.com
}

Received 27 March 2014; Accepted 1 October 2014; Published 30 November 2014

Academic Editor: George L. Karakostas

Copyright (C) 2014 B. Deshpande and A. Handa. This is an open access article distributed under the Creative Commons Attribution License, which permits unrestricted use, distribution, and reproduction in any medium, provided the original work is properly cited.

We introduce the concept of (EA) property and occasional $w$-compatibility for hybrid pair $F: X \times X \rightarrow 2^{X}$ and $f: X \rightarrow X$. We also introduce common (EA) property for two hybrid pairs $F, G: X \rightarrow 2^{X}$ and $f, g: X \rightarrow X$. We establish some common coupled fixed point theorems for two hybrid pairs of mappings under $\varphi-\psi$ contraction on noncomplete metric spaces. An example is also given to validate our results. We improve, extend and generalize several known results. The results of this paper generalize the common fixed point theorems for hybrid pairs of mappings and essentially contain fixed point theorems for hybrid pair of mappings.

\section{Introduction and Preliminaries}

Let $(X, d)$ be a metric space and let $\mathrm{CB}(X)$ be the set of all nonempty closed bounded subsets of $X$. Let $D(x, A)$ denote the distance from $x$ to $A \subset X$ and let $H$ denote the Hausdorff metric induced by $d$; that is,

$$
\begin{array}{r}
D(x, A)=\inf _{a \in A} d(x, a), \\
H(A, B)=\max \left\{\sup _{a \in A} D(a, B), \sup _{b \in B} D(b, A)\right\}, \\
\forall A, B \in \mathrm{CB}(X) .
\end{array}
$$

The study of fixed points for multivalued contractions and nonexpansive mappings using the Hausdorff metric was initiated by Markin [1]. The existence of fixed points for various multivalued contractive mappings has been studied by many authors under different conditions. The theory of multivalued mappings has application in control theory, convex optimization, differential inclusions, and economics. In 1969, Nadler [2] extended the famous Banach contraction principle [3] from single-valued mapping to multivalued mapping and proved the fixed point theorem for the multivalued contraction. Many authors proved fixed point theorems for hybrid pair of mappings without assuming the continuity of any mapping involved including [4-7].

In [8], Gnana Bhaskar and Lakshmikantham established some coupled fixed point theorems and applied these results to study the existence and uniqueness of solution for periodic boundary value problems. Luong and Thuan [9] generalized the results of Gnana Bhaskar and Lakshmikantham [8]. Berinde [10] extended the results of Gnana Bhaskar and Lakshmikantham [8] and Luong and Thuan [9]. Lakshmikantham and Ćirić [11] proved coupled coincidence and common coupled fixed point theorems for nonlinear contractive mappings in partially ordered complete metric spaces and extended the results of Gnana Bhaskar and Lakshmikantham [8]. Jain et al. [12] extended and generalized the results of Berinde [10], Gnana Bhaskar and Lakshmikantham [8], Lakshmikantham and Ćirić [11], and Luong and Thuan [9].

Deshpande and Handa [13] generalized and intuitionistically fuzzified the results of Gnana Bhaskar and Lakshmikantham [8], Lakshmikantham and Ćirić [11], and Luong and Thuan [9], while Deshpande et al. [14] generalized and intuitionistically fuzzified the results of Berinde [10], Gnana Bhaskar and Lakshmikantham [8], Lakshmikantham and Ćirić [11], and Luong and Thuan [9]. In [15], Deshpande et al. proved a common coupled fixed point theorem for mappings 
under $\varphi$-contractive conditions on intuitionistic fuzzy metric spaces. As an application, the existence and uniqueness of solution to a nonlinear Fredholm integral equation have been studied.

Recently Samet et al. [16] claimed that most of the coupled fixed point theorems in the setting of single valued mappings on ordered metric spaces are consequences of well-known fixed point theorems.

These concepts were extended by Abbas et al. [17] to multivalued mappings and who obtained coupled coincidence point and common coupled fixed point theorems involving hybrid pair of mappings satisfying generalized contractive conditions in complete metric spaces. Very few authors studied coupled fixed point theorems for hybrid pair of mappings including [17-20].

In [17], Abbas et al. introduced the following concept.

Definition 1. Let $X$ be a nonempty set, $F: X \times X \rightarrow 2^{X}$ (a collection of all nonempty subsets of $X$ ), and let $g$ be a selfmapping on $X$. An element $(x, y) \in X \times X$ is called

(1) a coupled coincidence point of hybrid pair $\{F, g\}$ if $g(x) \in F(x, y)$ and $g(y) \in F(y, x)$,

(2) a common coupled fixed point of hybrid pair $\{F, g\}$ if $x=g(x) \in F(x, y)$ and $y=g(y) \in F(y, x)$.

We denote the set of coupled coincidence points of mappings $F$ and $g$ by $C\{F, g\}$. Note that if $(x, y) \in C\{F, g\}$, then $(y, x)$ is also in $C\{F, g\}$.

Definition 2. Let $F: X \times X \rightarrow 2^{X}$ be a multivalued mapping and let $g$ be a self-mapping on $X$. The hybrid pair $\{F, g\}$ is called $w$-compatible if $g(F(x, y)) \subseteq F(g x, g y)$ whenever $(x, y) \in C\{F, g\}$.

Definition 3. Let $F: X \times X \rightarrow 2^{X}$ be a multivalued mapping and let $g$ be a self-mapping on $X$. The mapping $g$ is called $F$-weakly commuting at some point $(x, y) \in X \times X$ if $g^{2} x \in$ $F(g x, g y)$ and $g^{2} y \in F(g y, g x)$.

Aamri and El Moutawakil [21] defined (EA) property for self-mappings which contained the class of noncompatible mappings. Kamran [22] extended the (EA) property for hybrid pair $f: X \rightarrow X$ and $T: X \rightarrow 2^{X}$. Liu et al. [23] introduced common (EA) property for hybrid pairs of single and multivalued mappings and gave some new common fixed point theorems under hybrid contractive conditions. Abbas and Rhoades [24] extended the concept of occasionally weakly compatible mappings for hybrid pair $f: X \rightarrow X$ and $T: X \rightarrow 2^{X}$.

In this paper, we introduce the concept of (EA) property and occasional $w$-compatibility for hybrid pair $F: X \times$ $X \rightarrow 2^{X}$ and $f: X \rightarrow X$. We also introduce common (EA) property for two hybrid pairs $F, G: X \times X \rightarrow 2^{X}$ and $f, g: X \rightarrow X$. We establish some common coupled fixed point theorems for two hybrid pairs of mappings under $\varphi-\psi$ contraction on noncomplete metric spaces. The $\varphi-\psi$ contraction is weaker contraction than the contraction defined in Gnana Bhaskar and Lakshmikantham [8] and Luong and Thuan [9]. We improve, extend, and generalize the results of Berinde [10], Gnana Bhaskar and Lakshmikantham [8], Jain et al. [12], Lakshmikantham and Ćirić [11], Liu et al. [23], and Luong and Thuan [9]. The results of this paper generalize the common fixed point theorems for hybrid pairs of mappings and essentially contain fixed point theorems for hybrid pair of mappings.

\section{Main Results}

We first define the following.

Definition 4. Mappings $f: X \rightarrow X$ and $F: X \times X \rightarrow \mathrm{CB}(X)$ are said to satisfy the (EA) property if there exist sequences $\left\{x_{n}\right\},\left\{y_{n}\right\}$ in $X$, some $u, v$ in $X$, and $A, B$ in $\mathrm{CB}(X)$ such that

$$
\begin{aligned}
& \lim _{n \rightarrow \infty} f x_{n}=u \in A=\lim _{n \rightarrow \infty} F\left(x_{n}, y_{n}\right), \\
& \lim _{n \rightarrow \infty} f y_{n}=v \in B=\lim _{n \rightarrow \infty} F\left(y_{n}, x_{n}\right) .
\end{aligned}
$$

Definition 5. Let $f, g: X \rightarrow X$ and $F, G: X \times X \rightarrow \mathrm{CB}(X)$. The pairs $\{F, f\}$ and $\{G, g\}$ are said to satisfy the common (EA) property if there exist sequences $\left\{x_{n}\right\},\left\{y_{n}\right\},\left\{u_{n}\right\}$, and $\left\{v_{n}\right\}$ in $X$, some $u, v$ in $X$, and $A, B, C, D$ in $\mathrm{CB}(X)$ such that

$$
\begin{gathered}
\lim _{n \rightarrow \infty} F\left(x_{n}, y_{n}\right)=A, \quad \lim _{n \rightarrow \infty} G\left(u_{n}, v_{n}\right)=B, \\
\lim _{n \rightarrow \infty} f x_{n}=\lim _{n \rightarrow \infty} g u_{n}=u \in A \cap B, \\
\lim _{n \rightarrow \infty} F\left(y_{n}, x_{n}\right)=C, \quad \lim _{n \rightarrow \infty} G\left(v_{n}, u_{n}\right)=D, \\
\lim _{n \rightarrow \infty} f y_{n}=\lim _{n \rightarrow \infty} g v_{n}=v \in C \cap D .
\end{gathered}
$$

Example 6. Let $X=[1,+\infty)$ with the usual metric. Define $f, g: X \rightarrow X$ and $F, G: X \times X \rightarrow \mathrm{CB}(X)$ by

$$
\begin{gathered}
F(x, y)=[2,3+2 x+y], \quad G(x, y)=\left[2,3+\frac{3 x+y}{4}\right] \\
f(x)=2+x, \quad g(x)=1+\frac{x}{2}, \quad \forall x, y \in X .
\end{gathered}
$$

Consider the sequences

$$
\begin{array}{ll}
\left\{x_{n}\right\}=\left\{2+\frac{1}{n}\right\}, \quad\left\{y_{n}\right\}=\left\{4+\frac{1}{n}\right\}, \\
\left\{u_{n}\right\}=\left\{6+\frac{1}{n}\right\}, \quad\left\{v_{n}\right\}=\left\{10+\frac{1}{n}\right\} .
\end{array}
$$


Clearly,

$$
\begin{gathered}
\lim _{n \rightarrow \infty} F\left(x_{n}, y_{n}\right)=[2,11]=A, \\
\lim _{n \rightarrow \infty} G\left(u_{n}, v_{n}\right)=[2,10]=B, \\
\lim _{n \rightarrow \infty} f x_{n}=\lim _{n \rightarrow \infty} g u_{n}=4 \in A \cap B, \\
\lim _{n \rightarrow \infty} F\left(y_{n}, x_{n}\right)=[2,13]=C, \\
\lim _{n \rightarrow \infty} G\left(v_{n}, u_{n}\right)=[2,12]=D, \\
\lim _{n \rightarrow \infty} f y_{n}=\lim _{n \rightarrow \infty} g v_{n}=6 \in C \cap D .
\end{gathered}
$$

Therefore, the pairs $\{F, f\}$ and $\{G, g\}$ are said to satisfy the common (EA) property.

Definition 7. Mappings $F: X \times X \rightarrow 2^{X}$ and $f: X \rightarrow X$ are said to be occasionally $w$-compatible if and only if there exists some point $(x, y) \in X \times X$ such that $f x \in F(x, y), f y \in$ $F(y, x)$, and $f F(x, y) \subseteq F(f x, f y)$.

Example 8. Let $X=[0,+\infty)$ with usual metric. Define $f$ : $X \rightarrow X, F: X \times X \rightarrow \mathrm{CB}(X)$ by

$$
\begin{gathered}
f x= \begin{cases}0, & 0 \leq x<1, \\
4 x, & 1 \leq x<\infty,\end{cases} \\
F(x, y)= \begin{cases}{[0,1+2 x+y],} & (x, y) \neq(0,0), \\
\{x\}, & (x, y)=(0,0) .\end{cases}
\end{gathered}
$$

It can be easily verified that $(0,0)$ and $(1,1)$ are coupled coincidence points of $f$ and $F$, but $f F(0,0) \subseteq F(f 0, f 0)$ and $f F(1,1) \nsubseteq F(f 1, f 1)$. So $f$ and $F$ are not $w$-compatible. However, the pair $\{F, f\}$ is occasionally $w$-compatible.

Let $\Phi$ denote the set of all functions $\varphi:[0,+\infty) \rightarrow$ $[0,+\infty)$ satisfying the following:

$$
\begin{aligned}
& \left(\mathrm{i}_{\varphi}\right) \varphi \text { is continuous and strictly increasing, } \\
& \left(\mathrm{ii}_{\varphi}\right) \varphi(t)<t \text { for all } t>0, \\
& \left(\mathrm{iii}_{\varphi}\right) \varphi(t+s) \leq \varphi(t)+\varphi(s) \text { for all } t, s>0 .
\end{aligned}
$$

And let $\Psi$ denote the set of all functions $\psi:[0,+\infty) \rightarrow$ $[0,+\infty)$ which satisfies

$$
\begin{aligned}
& \left(\mathrm{i}_{\psi}\right) \lim _{t \rightarrow r} \psi(t)>0 \text { for all } r>0 \text { and } \lim _{t \rightarrow 0+} \psi(t)= \\
& 0, \\
& \left(\mathrm{ii}_{\psi}\right) \psi(t)>0 \text { for all } t>0 \text { and } \psi(0)=0 .
\end{aligned}
$$

Note that, by $\left(\mathrm{i}_{\varphi}\right)$ and $\left(\mathrm{ii}_{\varphi}\right)$, we have that $\varphi(t)=0$ if and only if $t=0$. For example, functions $\varphi_{1}(t)=k t$ where $k>$ $0, \varphi_{2}(t)=t /(t+1), \varphi_{3}(t)=\ln (t+1)$, and $\varphi_{4}(t)=\min \{t, 1\}$ are in $\Phi, \psi_{1}(t)=k t$ where $k>0, \psi_{2}(t)=(\ln (2 t+1)) / 2$, and $\psi_{3}(t)=\left\{\begin{array}{ll}1, & t=0 \\ t /(t+1), & 0<t<1 \\ 1, & t=1 \\ t / 2, & t>1\end{array}\right.$ are in $\Psi$.

Now, we prove our main results.
Theorem 9. Let $(X, d)$ be a metric space. Assume $F, G: X \times$ $X \rightarrow C B(X)$ and $f, g: X \rightarrow X$ to be mappings satisfying the following.

(1) $\{F, f\}$ and $\{G, g\}$ satisfy the common (EA) property.

(2) For all $x, y, u, v \in X$, there exist some $\varphi \in \Phi$ and some $\psi \in \Psi$ such that

$$
\begin{aligned}
\varphi( & \left.\frac{H(F(x, y), G(u, v))+H(F(y, x), G(v, u))}{2}\right) \\
\leq & \varphi\left(\frac{d(f x, g u)+d(f y, g v)}{2}\right) \\
& -\psi\left(\frac{d(f x, g u)+d(f y, g v)}{2}\right) .
\end{aligned}
$$

(3) $f(X)$ and $g(X)$ are closed subsets of $X$. Then

(a) $F$ and $f$ have a coupled coincidence point,

(b) $G$ and $g$ have a coupled coincidence point,

(c) $F$ and $f$ have a common coupled fixed point, if $f$ is F-weakly commuting at $(x, y)$ and $f^{2} x=f x$ and $f^{2} y=f y$ for $(x, y) \in C\{F, f\}$,

(d) $G$ and $g$ have a common coupled fixed point, if $g$ is $G$-weakly commuting at $(\tilde{x}, \tilde{y})$ and $g^{2} \tilde{x}=g \tilde{x}$ and $g^{2} \tilde{y}=g \tilde{y}$ for $(\tilde{x}, \tilde{y}) \in C\{G, g\}$,

(e) $F, G, f$, and $g$ have common coupled fixed point provided that both (c) and (d) are true.

Proof. Since $\{F, f\}$ and $\{G, g\}$ satisfy the common (EA) property, there exist sequences $\left\{x_{n}\right\},\left\{y_{n}\right\},\left\{u_{n}\right\}$, and $\left\{v_{n}\right\}$ in $X$, some $u, v$ in $X$, and $A, B, C, D$ in $\mathrm{CB}(X)$ such that

$$
\begin{aligned}
\lim _{n \rightarrow \infty} F\left(x_{n}, y_{n}\right) & =A, \quad \lim _{n \rightarrow \infty} G\left(u_{n}, v_{n}\right)=B, \\
\lim _{n \rightarrow \infty} f x_{n} & =\lim _{n \rightarrow \infty} g u_{n}=u \in A \cap B, \\
\lim _{n \rightarrow \infty} F\left(y_{n}, x_{n}\right) & =C, \quad \lim _{n \rightarrow \infty} G\left(v_{n}, u_{n}\right)=D, \\
\lim _{n \rightarrow \infty} f y_{n} & =\lim _{n \rightarrow \infty} g v_{n}=v \in C \cap D .
\end{aligned}
$$

Since $f(X)$ and $g(X)$ are closed subsets of $X$, then there exist $x, y, \tilde{x}, \tilde{y} \in X$,

$$
u=f x=g \tilde{x}, \quad v=f y=g \tilde{y} .
$$

Now, by using condition (2) of Theorem 9, we get

$$
\begin{aligned}
\varphi( & \left.\frac{H\left(F(x, y), G\left(u_{n}, v_{n}\right)\right)+H\left(F(y, x), G\left(v_{n}, u_{n}\right)\right)}{2}\right) \\
\leq & \varphi\left(\frac{d\left(f x, g u_{n}\right)+d\left(f y, g v_{n}\right)}{2}\right) \\
& -\psi\left(\frac{d\left(f x, g u_{n}\right)+d\left(f y, g v_{n}\right)}{2}\right) .
\end{aligned}
$$


Letting $n \rightarrow \infty$ in the above inequality, by using (9), (10), $\left(\mathrm{i}_{\varphi}\right),\left(\mathrm{ii}_{\varphi}\right)$, and $\left(\mathrm{i}_{\psi}\right)$, we obtain

$$
\begin{gathered}
\varphi\left(\frac{H(F(x, y), B)+H(F(y, x), D)}{2}\right) \\
\leq \varphi(0)-0=0-0=0,
\end{gathered}
$$

which, by $\left(\mathrm{i}_{\varphi}\right)$ and $\left(\mathrm{ii}_{\varphi}\right)$, implies

$$
H(F(x, y), B)=0, \quad H(F(y, x), D)=0 .
$$

Since $f x \in B$ and $f y \in D$, it follows that

$$
f x \in F(x, y), \quad f y \in F(y, x) .
$$

That is, $(x, y)$ is a coupled coincidence point of $F$ and $f$. This proves (a). Again, by using condition (2) of Theorem 9, we get

$$
\begin{aligned}
\varphi( & \left.\frac{H\left(F\left(x_{n}, y_{n}\right), G(\tilde{x}, \tilde{y})\right)+H\left(F\left(y_{n}, x_{n}\right), G(\tilde{y}, \tilde{x})\right)}{2}\right) \\
\leq & \varphi\left(\frac{d\left(f x_{n}, g \widetilde{x}\right)+d\left(f y_{n}, g \widetilde{y}\right)}{2}\right) \\
& -\psi\left(\frac{d\left(f x_{n}, g \tilde{x}\right)+d\left(f y_{n}, g \widetilde{y}\right)}{2}\right) .
\end{aligned}
$$

Letting $n \rightarrow \infty$ in the above inequality, by using (9), (10), $\left(\mathrm{i}_{\varphi}\right),\left(\mathrm{ii}_{\varphi}\right)$, and $\left(\mathrm{i}_{\psi}\right)$, we obtain

$$
\begin{gathered}
\varphi\left(\frac{H(A, G(\tilde{x}, \tilde{y}))+H(C, G(\tilde{y}, \tilde{x}))}{2}\right) \\
\leq \varphi(0)-0=0-0=0,
\end{gathered}
$$

which, by $\left(\mathrm{i}_{\varphi}\right)$ and $\left(\mathrm{ii}_{\varphi}\right)$, implies

$$
H(A, G(\tilde{x}, \tilde{y}))=0, \quad H(C, G(\tilde{y}, \tilde{x}))=0 .
$$

Since $g \tilde{x} \in A$ and $g \tilde{y} \in C$, it follows that

$$
g \tilde{x} \in G(\tilde{x}, \tilde{y}), \quad g \tilde{y} \in G(\tilde{y}, \tilde{x}) .
$$

That is, $(\widetilde{x}, \tilde{y})$ is a coupled coincidence point of $G$ and $g$. This proves (b).

Furthermore, from condition (c), we have $f$ which is $F$ weakly commuting at $(x, y)$; that is, $f^{2} x \in F(f x, f y), f^{2} y \in$ $F(f y, f x)$ and $f^{2} x=f x, f^{2} y=f y$. Thus, $f x=f^{2} x \in$ $F(f x, f y)$ and $f y=f^{2} y \in F(f y, f x)$; that is, $u=f u \in F(u, v)$ and $v=f v \in F(v, u)$. This proves (c). A similar argument proves (d). Then (e) holds immediately.

Put $f=g$ in Theorem 9, and we get the following result.

Corollary 10. Let $(X, d)$ be a metric space. Assume $F, G: X \times$ $X \rightarrow C B(X)$ and $g: X \rightarrow X$ to be mappings such that
(1) $\{F, g\}$ and $\{G, g\}$ satisfy the common (EA) property,

(2) for all $x, y, u, v \in X$, there exist some $\varphi \in \Phi$ and some $\psi \in \Psi$ such that

$$
\begin{aligned}
\varphi( & \left.\frac{H(F(x, y), G(u, v))+H(F(y, x), G(v, u))}{2}\right) \\
\leq & \varphi\left(\frac{d(g x, g u)+d(g y, g v)}{2}\right) \\
& -\psi\left(\frac{d(g x, g u)+d(g y, g v)}{2}\right),
\end{aligned}
$$

(3) $g(X)$ is a closed subset of $X$. Then

(a) F and $g$ have a coupled coincidence point,

(b) $G$ and $g$ have a coupled coincidence point,

(c) $F$ and $g$ have a common coupled fixed point, if $g$ is F-weakly commuting at $(x, y)$ and $g^{2} x=g x$ and $g^{2} y=$ gy for $(x, y) \in C\{F, g\}$,

(d) $G$ and $g$ have a common coupled fixed point, if $g$ is G-weakly commuting at $(\widetilde{x}, \tilde{y})$ and $g^{2} \widetilde{x}=g \widetilde{x}$ and $g^{2} \tilde{y}=g \tilde{y}$ for $(\tilde{x}, \tilde{y}) \in C\{G, g\}$,

(e) $F, G$, and $g$ have common coupled fixed point provided that both (c) and (d) are true.

Put $F=G$ and $f=g$ in Theorem 9, and we get the following result.

Corollary 11. Let $(X, d)$ be a metric space. Assume $F: X \times$ $X \rightarrow C B(X)$ and $g: X \rightarrow X$ to be mappings such that

(1) $\{F, g\}$ satisfies the (EA) property,

(2) for all $x, y, u, v \in X$, there exist some $\varphi \in \Phi$ and some $\psi \in \Psi$ such that

$$
\begin{gathered}
\varphi\left(\frac{H(F(x, y), F(u, v))+H(F(y, x), F(v, u))}{2}\right) \\
\leq \varphi\left(\frac{d(g x, g u)+d(g y, g v)}{2}\right) \\
\quad-\psi\left(\frac{d(g x, g u)+d(g y, g v)}{2}\right) .
\end{gathered}
$$

If (3) of Corollary 10 holds. Then

(a) F and $g$ have a coupled coincidence point,

(b) $F$ and $g$ have a common coupled fixed point, if $g$ is $F$ weakly commuting at $(x, y)$ and $g^{2} x=g x$ and $g^{2} y=$ gy for $(x, y) \in C\{F, g\}$.

Corollary 12. Let $(X, d)$ be a metric space. Assume $F, G: X \times$ $X \rightarrow C B(X)$ and $f, g: X \rightarrow X$ to be mappings satisfying (1) of Theorem 9 and 
(1) for all $x, y, u, v \in X$, there exists some $\psi \in \Psi$ such that

$$
\begin{gathered}
H(F(x, y), G(u, v))+H(F(y, x), G(v, u)) \\
\leq d(f x, g u)+d(f y, g v) \\
\quad-2 \psi\left(\frac{d(f x, g u)+d(f y, g v)}{2}\right) .
\end{gathered}
$$

If (3) of Theorem 9 holds, then

(a) F and $f$ have a coupled coincidence point,

(b) $G$ and $g$ have a coupled coincidence point,

(c) $F$ and $f$ have a common coupled fixed point, if $f$ is $F$ weakly commuting at $(x, y)$ and $f^{2} x=f x$ and $f^{2} y=$ fy for $(x, y) \in C\{F, f\}$,

(d) $G$ and $g$ have a common coupled fixed point, if $g$ is $G$ weakly commuting at $(\tilde{x}, \tilde{y})$ and $g^{2} \tilde{x}=g \tilde{x}$ and $g^{2} \tilde{y}=$ $g \tilde{y}$ for $(\tilde{x}, \tilde{y}) \in C\{G, g\}$,

(e) $F, G, f$, and $g$ have common coupled fixed point provided that both (c) and (d) are true.

Proof. If $\psi \in \Psi$, then for all $r>0, r \psi \in \Psi$. Now divide condition (1) of Corollary 12 by 4 and take $\varphi(t)=(1 / 2) t, t \in$ $[0,+\infty)$, and then the above condition reduces to condition (2) of Theorem 9 with $\psi_{1}=(1 / 2) \psi$ and hence by Theorem 9 we get Corollary 12.

Put $f=g$ in Corollary 12 , and we get the following result.

Corollary 13. Let $(X, d)$ be a metric space. Assume $F, G: X \times$ $X \rightarrow C B(X)$ and $g: X \rightarrow X$ to be mappings satisfying (1) of Corollary 10 and

(1) for all $x, y, u, v \in X$, there exists some $\psi \in \Psi$ such that

$$
\begin{gathered}
H(F(x, y), G(u, v))+H(F(y, x), G(v, u)) \\
\leq d(g x, g u)+d(g y, g v) \\
\quad-2 \psi\left(\frac{d(g x, g u)+d(g y, g v)}{2}\right) .
\end{gathered}
$$

If (3) of Corollary 10 holds, then

(a) F and $g$ have a coupled coincidence point,

(b) $G$ and $g$ have a coupled coincidence point,

(c) $F$ and $g$ have a common coupled fixed point, if $g$ is $F$ weakly commuting at $(x, y)$ and $g^{2} x=g x$ and $g^{2} y=$ gy for $(x, y) \in C\{F, g\}$,

(d) $G$ and $g$ have a common coupled fixed point, if $g$ is $G$ weakly commuting at $(\tilde{x}, \tilde{y})$ and $g^{2} \tilde{x}=g \tilde{x}$ and $g^{2} \tilde{y}=$ $g \tilde{y}$ for $(\tilde{x}, \tilde{y}) \in C\{G, g\}$,

(e) F, G, and $g$ have common coupled fixed point provided that both (c) and (d) are true. result.

Put $F=G$ and $f=g$ in Corollary 12, we get the following
Corollary 14. Let $(X, d)$ be a metric space. Assume $F: X \times$ $X \rightarrow C B(X)$ and $g: X \rightarrow X$ to be mappings satisfying (1) of Corollary 11 and

(1) for all $x, y, u, v \in X$, there exists some $\psi \in \Psi$ such that

$$
\begin{aligned}
& H(F(x, y), F(u, v))+H(F(y, x), F(v, u)) \\
& \leq d(g x, g u)+d(g y, g v) \\
& \quad-2 \psi\left(\frac{d(g x, g u)+d(g y, g v)}{2}\right) .
\end{aligned}
$$

If (3) of Corollary 10 holds, then

(a) $F$ and $g$ have a coupled coincidence point,

(b) $F$ and $g$ have a common coupled fixed point, if $g$ is $F$ weakly commuting at $(x, y)$ and $g^{2} x=g x$ and $g^{2} y=$ gy for $(x, y) \in C\{F, g\}$.

Theorem 15. Let $(X, d)$ be a metric space. Assume $F, G: X \times$ $X \rightarrow C B(X)$ and $f, g: X \rightarrow X$ to be mappings satisfying (1) of Theorem 9 and (2) of Theorem 9 and

(1) $\{F, f\}$ and $\{G, g\}$ are $w$-compatible.

(2) Suppose that either

(a) $g(X)$ is a closed subset of $X$ and $G(X \times X) \subseteq f(X)$ or

(b) $f(X)$ is a closed subset of $X$ and $F(X \times X) \subseteq$ $g(X)$.

Then F, G, $f$, and g have a common coupled fixed point.

Proof. Since $\{F, f\}$ and $\{G, g\}$ satisfy the common (EA) property, there exist sequences $\left\{x_{n}\right\},\left\{y_{n}\right\},\left\{u_{n}\right\}$, and $\left\{v_{n}\right\}$ in $X$, some $u, v$ in $X$, and $A, B, C, D$ in $\mathrm{CB}(X)$ satisfying (9). Suppose (a) holds; that is, $g(X)$ is a closed subset of $X$, and then there exist $\tilde{x}, \tilde{y} \in X$, and we have

$$
u=g \tilde{x}, \quad v=g \tilde{y} .
$$

As in Theorem 9, we can prove that

$$
g \tilde{x} \in G(\tilde{x}, \tilde{y}), \quad g \tilde{y} \in G(\tilde{y}, \tilde{x}) .
$$

That is, $(\tilde{x}, \tilde{y})$ is a coupled coincidence point of $G$ and $g$. Hence, $(\tilde{x}, \tilde{y}) \in C\{G, g\}$. From $w$-compatibility of $\{G, g\}$, we have $g G(\tilde{x}, \tilde{y}) \subseteq G(g \tilde{x}, g \widetilde{y})$; hence, $g^{2} \tilde{x} \in G(g \tilde{x}, g \widetilde{y})$ and $g^{2} \tilde{y} \in G(g \tilde{y}, g \tilde{x})$; that is, $g u \in G(u, v)$ and $g v \in G(v, u)$. Now, we shall show that $u=g u$ and $v=g v$. Suppose, not. Then, by condition (2) of Theorem 9 , we get

$$
\begin{aligned}
\varphi( & \left.\frac{H\left(F\left(x_{n}, y_{n}\right), G(u, v)\right)+H\left(F\left(y_{n}, x_{n}\right), G(v, u)\right)}{2}\right) \\
\leq & \varphi\left(\frac{d\left(f x_{n}, g u\right)+d\left(f y_{n}, g v\right)}{2}\right) \\
& -\psi\left(\frac{d\left(f x_{n}, g u\right)+d\left(f y_{n}, g v\right)}{2}\right) .
\end{aligned}
$$


Letting $n \rightarrow \infty$ in the above inequality, by using $(9)$ and $\left(\mathrm{i}_{\varphi}\right)$, we obtain

$$
\begin{aligned}
\varphi( & \left.\frac{H(A, G(u, v))+H(C, G(v, u))}{2}\right) \\
\leq & \varphi\left(\frac{d(u, g u)+d(v, g v)}{2}\right) \\
& -\lim _{n \rightarrow \infty} \psi\left(\frac{d\left(f x_{n}, g u\right)+d\left(f y_{n}, g v\right)}{2}\right) .
\end{aligned}
$$

Since $u \in A, v \in C, g u \in G(u, v)$, and $g v \in G(v, u)$, therefore, by $\left(\mathrm{i}_{\psi}\right)$, we get

$$
\begin{aligned}
\varphi( & \left.\frac{d(u, g u)+d(v, g v)}{2}\right) \\
\leq & \varphi\left(\frac{H(A, G(u, v))+H(C, G(v, u))}{2}\right) \\
\leq & \varphi\left(\frac{d(u, g u)+d(v, g v)}{2}\right) \\
& -\lim _{n \rightarrow \infty} \psi\left(\frac{d\left(f x_{n}, g u\right)+d\left(f y_{n}, g v\right)}{2}\right) \\
< & \left(\frac{d(u, g u)+d(v, g v)}{2}\right),
\end{aligned}
$$

which is a contradiction. Thus, $u=g u$ and $v=g v$. Hence, we have

$$
u=g u \in G(u, v), \quad v=g v \in G(v, u) .
$$

Since $G(X \times X) \subseteq f(X)$, then there exist $x, y \in X$ such that $f x=u=g u \in G(u, v)$ and $f y=v=g v \in G(v, u)$. Now, by condition (2) of Theorem 9, $\left(\mathrm{i}_{\varphi}\right),\left(\mathrm{ii}_{\varphi}\right)$, and $\left(\mathrm{ii}_{\psi}\right)$, we get

$$
\begin{aligned}
\varphi( & \left.\frac{D(F(x, y), u)+D(F(y, x), v)}{2}\right) \\
\leq & \varphi\left(\frac{H(F(x, y), G(u, v))+H(F(y, x), G(v, u))}{2}\right) \\
\leq & \varphi\left(\frac{d(f x, g u)+d(f y, g v)}{2}\right) \\
& -\psi\left(\frac{d(f x, g u)+d(f y, g v)}{2}\right) \\
\leq & 0-0=0,
\end{aligned}
$$

which, by $\left(\mathrm{i}_{\varphi}\right)$ and $\left(\mathrm{ii}_{\varphi}\right)$, implies

$$
D(F(x, y), u)=D(F(y, x), v)=0 .
$$

Thus,

$$
u=f x \in F(x, y), \quad v=f y \in F(y, x) .
$$

That is, $(x, y)$ is a coupled coincidence point of $F$ and $f$. Hence, $(x, y) \in C\{F, f\}$. From $w$-compatibility of $\{F, f\}$, we have $f F(x, y) \subseteq F(f x, f y)$; hence $f^{2} x \in F(f x, f y)$ and $f^{2} y \in F(f y, f x)$; that is, $f u \in F(u, v)$ and $f v \in F(v, u)$. Now, we shall show that $f u=u$ and $f v=v$. Suppose, not. Then, by condition (2) of Theorem 9 and $\left(\mathrm{ii}_{\psi}\right)$, we get

$$
\begin{aligned}
\varphi( & \left.\frac{d(f u, u)+d(f v, v)}{2}\right) \\
\leq & \varphi\left(\frac{H(F(u, v), G(u, v))+H(F(v, u), G(v, u))}{2}\right) \\
& \leq \varphi\left(\frac{d(f u, g u)+d(f v, g v)}{2}\right) \\
& -\psi\left(\frac{d(f u, g u)+d(f v, g v)}{2}\right) \\
\leq & \left(\frac{d(f u, u)+d(f v, v)}{2}\right) \\
& -\psi\left(\frac{d(f u, u)+d(f v, v)}{2}\right) \\
< & \left(\frac{d(f u, u)+d(f v, v)}{2}\right)
\end{aligned}
$$

which is a contradiction. Thus, $f u=u$ and $f v=v$. Hence, we have

$$
u=f u \in F(u, v), \quad v=f v \in F(v, u) .
$$

Therefore, $(u, v)$ is a common coupled fixed point of the pairs $\{F, f\}$ and $\{G, g\}$. The proof is similar when (b) holds.

If we put $f=g$ in Theorem 15, we get the following result.

Corollary 16. Let $(X, d)$ be a metric space. Assume $F, G: X \times$ $X \rightarrow C B(X)$ and $g: X \rightarrow X$ to be mappings satisfying (1) of Corollary 10 and (2) of Corollary 10 and

(1) $\{F, g\}$ and $\{G, g\}$ are $w$-compatible;

(2) suppose that either

(a) $g(X)$ is a closed subset of $X$ and $G(X \times X) \subseteq g(X)$ or

(b) $g(X)$ is a closed subset of $X$ and $F(X \times X) \subseteq g(X)$.

Then F, G, and g have a common coupled fixed point.

If we put $F=G$ and $f=g$ in Theorem 15, we get the following result.

Corollary 17. Let $(X, d)$ be a metric space. Assume $F: X \times$ $X \rightarrow C B(X)$ and $g: X \rightarrow X$ to be mappings satisfying (1) of Corollary 11 and (2) of Corollary 11 and

(1) $\{F, g\}$ is w-compatible;

(2) $g(X)$ is a closed subset of $X$ and $F(X \times X) \subseteq g(X)$.

Then $F$ and $g$ have a common coupled fixed point. 
Corollary 18. Let $(X, d)$ be a metric space. Assume $F, G: X \times$ $X \rightarrow C B(X)$ and $f, g: X \rightarrow X$ to be mappings satisfying (1) of Theorem 9, (1) of Corollary 12, (1) of Theorem 15, and (2) of Theorem 15; then F, G, $f$, and $g$ have a common coupled fixed point.

Proof. If $\psi \in \Psi$, then for all $r>0, r \psi \in \Psi$. If we divide condition (1) of Corollary 12 by 4 and take $\varphi(t)=(1 / 2) t, t \in$ $[0,+\infty)$, then it reduces to condition (2) of Theorem 9 with $\psi_{1}=(1 / 2) \psi$ and hence by Theorem 15 we get Corollary 18 . result.

If we put $f=g$ in Corollary 18, we get the following

Corollary 19. Let $(X, d)$ be a metric space. Assume $F, G: X \times$ $X \rightarrow C B(X)$ and $g: X \rightarrow X$ to be mappings satisfying (1) of Corollary 10, (1) of Corollary 13, (1) of Corollary 16, and (2) of Corollary 16; then F, G, and $g$ have a common coupled fixed point.

If we put $F=G$ and $f=g$ in Corollary 18, we get the following result.

Corollary 20. Let $(X, d)$ be a metric space. Assume $F: X \times$ $X \rightarrow C B(X)$ and $g: X \rightarrow X$ to be mappings satisfying (1) of Corollary 11, (1) of Corollary 14, (1) of Corollary 17, and (2) of Corollary 17; then F and $g$ have a common coupled fixed point.

Theorem 21. Let $(X, d)$ be a metric space. Assume $F, G: X \times$ $X \rightarrow C B(X)$ and $f, g: X \rightarrow X$ to be mappings satisfying (2) of Theorem 9 and

(1) $\{F, f\}$ and $\{G, g\}$ are occasionally $w$-compatible.

Then $F, G, f$, and g have a common coupled fixed point.

Proof. Since the pairs $\{F, f\}$ and $\{G, g\}$ are occasionally $w$ compatible, therefore there exist some points $(x, y),(\widetilde{x}, \widetilde{y}) \in$ $X \times X$, such that

$$
\begin{aligned}
& f x \in F(x, y), \quad f y \in F(y, x), \quad f F(x, y) \subseteq F(f x, f y), \\
& g \tilde{x} \in G(\tilde{x}, \tilde{y}), \quad g \tilde{y} \in G(\tilde{y}, \tilde{x}), \quad g G(\tilde{x}, \tilde{y}) \subseteq G(g \tilde{x}, g \tilde{y}) .
\end{aligned}
$$

It follows that

$$
\begin{array}{ll}
f^{2} x \in F(f x, f y), & f^{2} y \in F(f y, f x), \\
g^{2} \tilde{x} \in G(g \tilde{x}, g \tilde{y}), & g^{2} \tilde{y} \in G(g \tilde{y}, g \tilde{x}) .
\end{array}
$$

Now, we shall show that $u=f x=g \tilde{x}$ and $v=f y=g \tilde{y}$. Suppose, not. Then, by condition (2) of Theorem 9 and ( $\mathrm{ii}_{\psi}$ ), we have

$$
\begin{aligned}
\varphi & \left(\frac{d(f x, g \tilde{x})+d(f y, g \tilde{y})}{2}\right) \\
& \leq \varphi\left(\frac{H(F(x, y), G(\tilde{x}, \tilde{y}))+H(F(y, x), G(\tilde{y}, \tilde{x}))}{2}\right)
\end{aligned}
$$

$$
\begin{aligned}
\leq & \varphi\left(\frac{d(f x, g \tilde{x})+d(f y, g \tilde{y})}{2}\right) \\
& -\psi\left(\frac{d(f x, g \tilde{x})+d(f y, g \tilde{y})}{2}\right) \\
< & \varphi\left(\frac{d(f x, g \tilde{x})+d(f y, g \tilde{y})}{2}\right),
\end{aligned}
$$

which is a contradiction. Thus, $f x=g \tilde{x}$ and $f y=g \tilde{y}$. Hence,

$$
u=f x=g \tilde{x}, \quad v=f y=g \tilde{y} .
$$

Thus, by (36), we get

$$
\begin{array}{ll}
f u \in F(u, v), & f v \in F(v, u), \\
g u \in G(u, v), & g v \in G(v, u) .
\end{array}
$$

Now, we shall show that $u=f u=g u$ and $v=f v=g v$. Suppose, not. Then, by condition (2) of Theorem 9 and $\left(\mathrm{ii}_{\psi}\right)$, we have

$$
\begin{aligned}
\varphi( & \left.\frac{d(f u, u)+d(f v, v)}{2}\right) \\
\leq & \varphi\left(\frac{H(F(u, v), G(\tilde{x}, \tilde{y}))+H(F(v, u), G(\tilde{y}, \tilde{x}))}{2}\right) \\
\leq & \varphi\left(\frac{d(f u, g \tilde{x})+d(f v, g \tilde{y})}{2}\right) \\
& -\psi\left(\frac{d(f u, g \tilde{x})+d(f v, g \tilde{y})}{2}\right) \\
\leq & \varphi\left(\frac{d(f u, u)+d(f v, v)}{2}\right) \\
& -\psi\left(\frac{d(f u, u)+d(f v, v)}{2}\right) \\
< & \varphi\left(\frac{d(f u, u)+d(f v, v)}{2}\right),
\end{aligned}
$$

which is a contradiction. Thus,

$$
u=f u, \quad v=f v .
$$

Similarly, we can show that

$$
u=g u, \quad v=g v .
$$

Thus, by (39), (41), and (42), we get

$$
\begin{array}{ll}
u=f u \in F(u, v), & v=f v \in F(v, u), \\
u=g u \in G(u, v), & v=g v \in G(v, u) .
\end{array}
$$

That is, $(u, v)$ is a common coupled fixed point of $F, G, f$, and g. 
Put $f=g$ in Theorem 21, and we get the following result.

Corollary 22. Let $(X, d)$ be a metric space. Assume $F, G: X \times$ $X \rightarrow C B(X)$ and $g: X \rightarrow X$ to be mappings satisfying (2) of Corollary 10 and

(1) $\{F, g\}$ and $\{G, g\}$ are occasionally $w$-compatible.

Then F, G, and $g$ have a common coupled fixed point.

Put $F=G$ and $f=g$ in Theorem 21, and we get the following result.

Corollary 23. Let $(X, d)$ be a metric space. Assume $F: X \times$ $X \rightarrow C B(X)$ and $g: X \rightarrow X$ to be mappings satisfying (2) of Corollary 11 and

(1) $\{F, g\}$ is occasionally w-compatible.

Then $F$ and $g$ have a common coupled fixed point.

Corollary 24. Let $(X, d)$ be a metric space. Assume $F, G: X \times$ $X \rightarrow C B(X)$ and $f, g: X \rightarrow X$ to be mappings satisfying (1) of Corollary 12 and (1) of Theorem 21; then F, G, f, and $g$ have a common coupled fixed point.

Proof. If $\psi \in \Psi$, then for all $r>0, r \psi \in \Psi$. If we divide condition (1) of Corollary 12 by 4 and take $\varphi(t)=(1 / 2) t, t \in$ $[0,+\infty)$, then it reduces to condition (2) of Theorem 9 with $\psi_{1}=(1 / 2) \psi$ and hence by Theorem 21 we get Corollary 24 .

Put $f=g$ in Corollary 24, and we get the following result.

Corollary 25. Let $(X, d)$ be a metric space. Assume $F, G: X \times$ $X \rightarrow C B(X)$ and $g: X \rightarrow X$ to be mappings satisfying (1) of Corollary 13 and (1) of Corollary 22; then F, G, and $g$ have a common coupled fixed point.

Put $F=G$ and $f=g$ in Corollary 24, and we get the following result.

Corollary 26. Let $(X, d)$ be a metric space. Assume $F: X \times$ $X \rightarrow C B(X)$ and $g: X \rightarrow X$ to be mappings satisfying (1) of Corollary 14 and (1) of Corollary 23; then F and $g$ have a common coupled fixed point.

Example 27. Suppose that $X=[0,1]$, equipped with the metric $d: X \times X \rightarrow[0,+\infty)$ defined as $d(x, y)=\max \{x, y\}$ and $d(x, x)=0$ for all $x, y \in X$. Let $F, G: X \times X \rightarrow \mathrm{CB}(X)$ be defined as

$$
\begin{aligned}
& F(x, y)=\left\{\begin{array}{ll}
\{0\}, & \text { for } x, y=1
\end{array}\right] \\
& G(x, y)= \begin{cases}\{0\}, & \text { for } x, y \in[0,1), \\
{\left[0, \frac{x+y}{8}\right],} & \text { for } x, y=1\end{cases}
\end{aligned}
$$

Suppose $f, g: X \rightarrow X$ be defined as

$$
\begin{aligned}
& f x=\left\{\begin{array}{ll}
x^{2}, & x \neq 1, \\
\frac{3}{2}, & x=1,
\end{array} \quad \forall x \in X\right. \\
& g x=\left\{\begin{array}{ll}
\frac{x}{2}, & x \neq 1, \\
1, & x=1,
\end{array} \quad \forall x \in X .\right.
\end{aligned}
$$

Define $\varphi:[0,+\infty) \rightarrow[0,+\infty)$ by

$$
\varphi(t)=\frac{t}{2}, \quad \forall t>0,
$$

and $\psi:[0,+\infty) \rightarrow[0,+\infty)$ by

$$
\psi(t)= \begin{cases}\frac{t}{4}, & \text { for } t \neq 1 \\ 1, & \text { for } t=1\end{cases}
$$

Now, for all $x, y, u, v \in X$ with $x, y, u, v \in[0,1)$, we have

Case (a). If $\left(x^{2}+y^{2}\right) / 4=(u+v) / 8$, then

$$
\begin{aligned}
\varphi( & \left.\frac{H(F(x, y), G(u, v))+H(F(y, x), G(v, u))}{2}\right) \\
= & \frac{1}{4}[H(F(x, y), G(u, v))+H(F(y, x), G(v, u))] \\
= & \frac{1}{4}\left[\frac{u+v}{8}+\frac{v+u}{8}\right] \\
\leq & \frac{1}{8}\left[\max \left\{x^{2}, \frac{u}{2}\right\}+\max \left\{y^{2}, \frac{v}{2}\right\}\right] \\
\leq & \frac{1}{8}[d(f x, g u)+d(f y, g v)] \\
\leq & \frac{1}{4}\left(\frac{d(f x, g u)+d(f y, g v)}{2}\right) \\
\leq & \varphi\left(\frac{d(f x, g u)+d(f y, g v)}{2}\right) \\
& -\psi\left(\frac{d(f x, g u)+d(f y, g v)}{2}\right) .
\end{aligned}
$$

Case (b). If $\left(x^{2}+y^{2}\right) / 4 \neq(u+v) / 8$ with $\left(x^{2}+y^{2}\right) / 4<(u+v) / 8$, then

$$
\begin{aligned}
\varphi( & \left.\frac{H(F(x, y), G(u, v))+H(F(y, x), G(v, u))}{2}\right) \\
& =\frac{1}{4}[H(F(x, y), G(u, v))+H(F(y, x), G(v, u))]
\end{aligned}
$$




$$
\begin{aligned}
= & \frac{1}{4}\left[\frac{u+v}{8}+\frac{v+u}{8}\right] \\
\leq & \frac{1}{8}\left[\max \left\{x^{2}, \frac{u}{2}\right\}+\max \left\{y^{2}, \frac{v}{2}\right\}\right] \\
\leq & \frac{1}{8}[d(f x, g u)+d(f y, g v)] \\
\leq & \frac{1}{4}\left(\frac{d(f x, g u)+d(f y, g v)}{2}\right) \\
\leq & \varphi\left(\frac{d(f x, g u)+d(f y, g v)}{2}\right) \\
& -\psi\left(\frac{d(f x, g u)+d(f y, g v)}{2}\right) .
\end{aligned}
$$

Similarly, we obtain the same result for $(u+v) / 8<\left(x^{2}+y^{2}\right) / 4$. Thus, the contractive condition (2) of Theorem 9 is satisfied for all $x, y, u, v \in X$ with $x, y, u, v \in[0,1)$. Again, for all $x, y, u, v \in X$ with $x, y \in[0,1)$ and $u, v=1$, we have

$$
\begin{aligned}
\varphi( & \left.\frac{H(F(x, y), G(u, v))+H(F(y, x), G(v, u))}{2}\right) \\
& =\frac{1}{4}[H(F(x, y), G(u, v))+H(F(y, x), G(v, u))] \\
& =\frac{1}{4}\left[\frac{x^{2}+y^{2}}{4}+\frac{y^{2}+x^{2}}{4}\right] \\
& \leq \frac{1}{8}\left[\max \left\{x^{2}, \frac{u}{2}\right\}+\max \left\{y^{2}, \frac{v}{2}\right\}\right] \\
& \leq \frac{1}{8}[d(f x, g u)+d(f y, g v)] \\
& \leq \frac{1}{4}\left(\frac{d(f x, g u)+d(f y, g v)}{2}\right) \\
& \leq \varphi\left(\frac{d(f x, g u)+d(f y, g v)}{2}\right) \\
& -\psi\left(\frac{d(f x, g u)+d(f y, g v)}{2}\right) .
\end{aligned}
$$

Thus, the contractive condition (2) of Theorem 9 is satisfied for all $x, y, u, v \in X$ with $x, y \in[0,1)$ and $u, v=$ 1. Similarly, we can see that the contractive condition (2) of Theorem 9 is satisfied for all $x, y, u, v \in X$ with $x, y, u, v=1$. Hence, the hybrid pairs $\{F, f\}$ and $\{G, g\}$ satisfy condition (2) of Theorem 9 , for all $x, y, u, v \in X$. In addition, all the other conditions of Theorem 9, Theorem 15, and Theorem 21 are satisfied and $z=(0,0)$ is a common coupled fixed point of $F, G, f$, and $g$.

\section{Conflict of Interests}

The authors declare that there is no conflict of interests regarding the publication of this paper.

\section{Acknowledgment}

The authors thank the referee for the extremely careful reading and very deep and useful comments and suggestions that contributed to the improvement of the paper.

\section{References}

[1] J. T. Markin, "Continuous dependence of fixed point sets," Proceedings of the American Mathematical Society, vol. 38, pp. 545-547, 1973.

[2] S. B. Nadler, "Multi-valued contraction mappings," Pacific Journal of Mathematics, vol. 30, pp. 475-488, 1969.

[3] S. Banach, "Sur les operations dans les ensembles abstraits et leur. Applications aux equations integrales," Fundamenta Mathematicae, vol. 3, pp. 133-181, 1922.

[4] B. Deshpande, "Common fixed point for set and single valued functions without continuity and compatibility," Mathematica Moravica, vol. 11, pp. 27-38, 2007.

[5] B. Deshpande and S. Chouhan, "Fixed points for two hybrid pairs of mappings satisfying some weaker conditions on noncomplete metric spaces," Southeast Asian Bulletin of Mathematics, vol. 35, no. 5, pp. 851-858, 2011.

[6] I. Kubiaczyk and B. Deshpande, "Noncompatibility, discontinuity in consideration of common fixed point of set and single valued maps," Southeast Asian Bulletin of Mathematics, vol. 32, no. 3, pp. 467-474, 2008.

[7] S. Sharma and B. Deshpande, "Fixed point theorems for set and single valued mappings without continuity and compatibility," Demonstratio Mathematica, vol. 40, no. 3, pp. 649-658, 2007.

[8] T. Gnana Bhaskar and V. Lakshmikantham, "Fixed point theorems in partially ordered metric spaces and applications," Nonlinear Analysis: Theory, Methods \& Applications, vol. 65, no. 7, pp. 1379-1393, 2006.

[9] N. V. Luong and N. X. Thuan, "Coupled fixed points in partially ordered metric spaces and application," Nonlinear Analysis. Theory, Methods \& Applications, vol. 74, no. 3, pp. 983-992, 2011.

[10] V. Berinde, "Coupled fixed point theorems for $\varphi$-contractive mixed monotone mappings in partially ordered metric spaces," Nonlinear Analysis: Theory, Methods \& Applications, vol. 75, no. 6, pp. 3218-3228, 2012.

[11] V. Lakshmikantham and L. Cirić, "Coupled fixed point theorems for nonlinear contractions in partially ordered metric spaces," Nonlinear Analysis: Theory, Methods \& Applications, vol. 70, no. 12, pp. 4341-4349, 2009.

[12] M. Jain, K. Tas, S. Kumar, and N. Gupta, "Coupled common fixed point results involving a $\varphi, \psi$-contractive condition for mixed $g$-monotone operators in partially ordered metric spaces," Journal of Inequalities and Applications, vol. 2012, article 285, 19 pages, 2012.

[13] B. Deshpande and A. Handa, "Nonlinear mixed monotonegeneralized contractions on partially ordered modified intuitionistic fuzzy metric spaces with application to integral equations," Afrika Matematika, 2013.

[14] B. Deshpande, S. Sharma, and A. Handa, " $\varphi$-contractive mixed monotone mappings in coupled fixed point consideration on 
intuitionistic fuzzy metric spaces with application to integral equations," The Journal of Fuzzy Mathematics, vol. 22, no. 1, pp. 401-418, 2014.

[15] B. Deshpande, S. Sharma, and A. Handa, "Common coupled fixed point theorems for nonlinear contractive condition on intuitionistic fuzzy metric spaces with application to integral equations," Journal of the Korean Society of Mathematical Education. Series B. The Pure and Applied Mathematics, vol. 20, no. 3, pp. 159-180, 2013.

[16] B. Samet, E. Karapinar, H. Aydi, and V. Ć. Rajić, "Discussion on some coupled fixed point theorems," Fixed Point Theory and Applications, vol. 2013, article 50, 2013.

[17] M. Abbas, L. Ćirić, B. Damjanović, and M. A. Khan, "Coupled coincidence and common fixed point theorems for hybrid pair of mappings," Fixed Point Theory and Applications, vol. 2012, article 4, 2012.

[18] M. Abbas, B. Ali, and A. Amini-Harandi, "Common fixed point theorem for a hybrid pair of mappings in Hausdorff fuzzy metric spaces," Fixed Point Theory and Applications, vol. 2012, article 225, 12 pages, 2012.

[19] W. Long, S. Shukla, and S. Radenovic, "Some coupled coincidence and common fixed point results for hybrid pair of mappings in 0-complete partial metric spaces," Fixed Point Theory and Applications, vol. 2013, article 145, 2013.

[20] N. Singh and R. Jain, "Coupled coincidence and common fixed point theorems for set-valued and single-valued mappings in fuzzy metric space," Journal of Fuzzy Set Valued Analysis, vol. 2012, Article ID jfsva-00129, 10 pages, 2012.

[21] M. Aamri and D. El Moutawakil, "Some new common fixed point theorems under strict contractive conditions," Journal of Mathematical Analysis and Applications, vol. 270, no. 1, pp. 181188, 2002.

[22] T. Kamran, "Coincidence and fixed points for hybrid strict contractions," Journal of Mathematical Analysis and Applications, vol. 299, no. 1, pp. 235-241, 2004.

[23] Y. Liu, J. Wu, and Z. Li, "Common fixed points of single-valued and multivalued maps," International Journal of Mathematics and Mathematical Sciences, vol. 2005, no. 19, pp. 3045-3055, 2005.

[24] M. Abbas and B. E. Rhoades, "Common fixed point theorems for hybrid pairs of occasionally weakly compatible mappings satisfying generalized contractive condition of integral type," Fixed Point Theory and Applications, vol. 2007, Article ID 054101, 9 pages, 2007. 


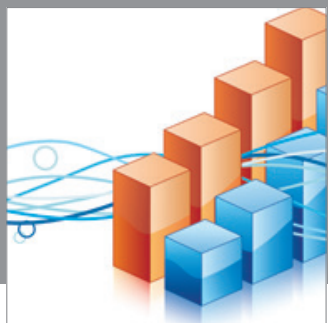

Advances in

Operations Research

mansans

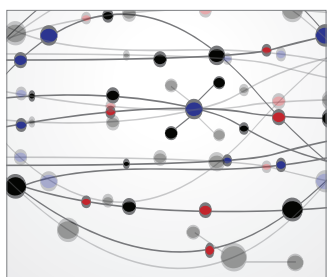

The Scientific World Journal
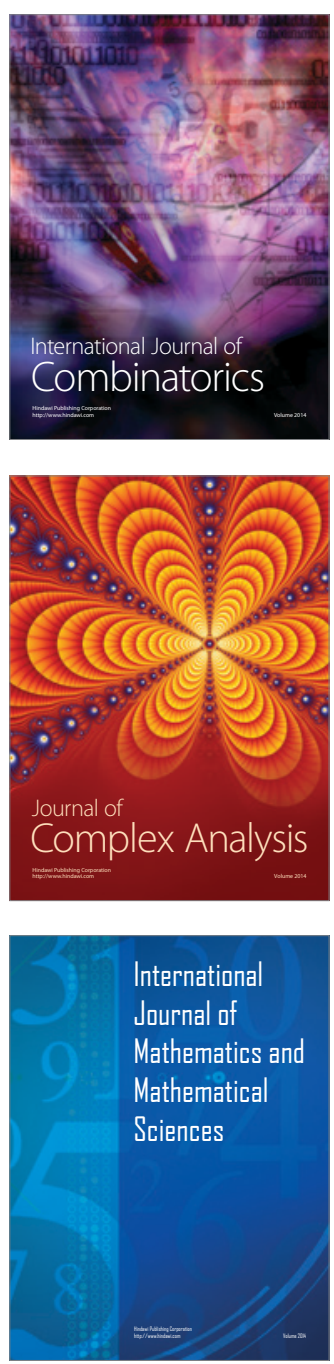
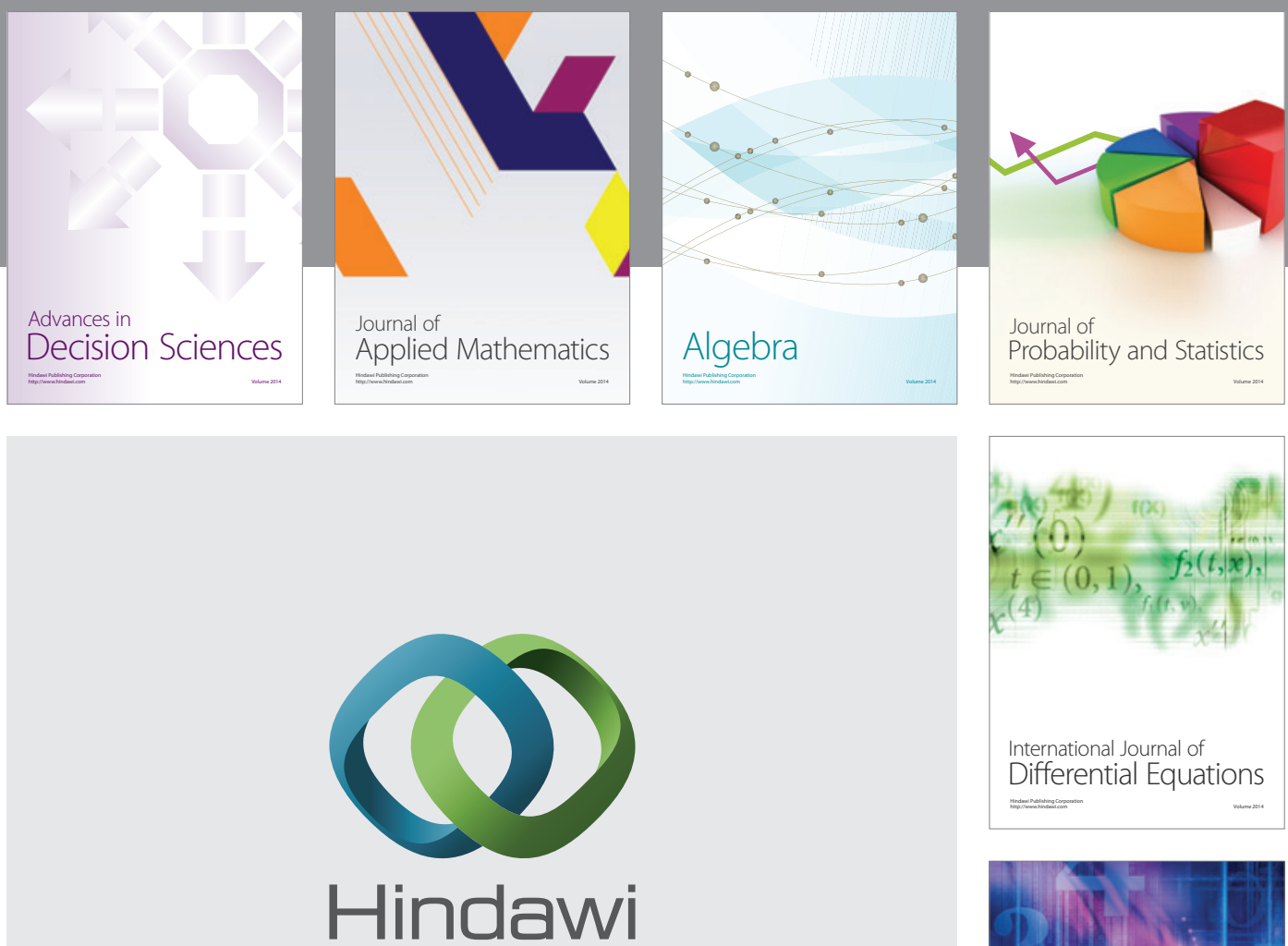

Submit your manuscripts at http://www.hindawi.com
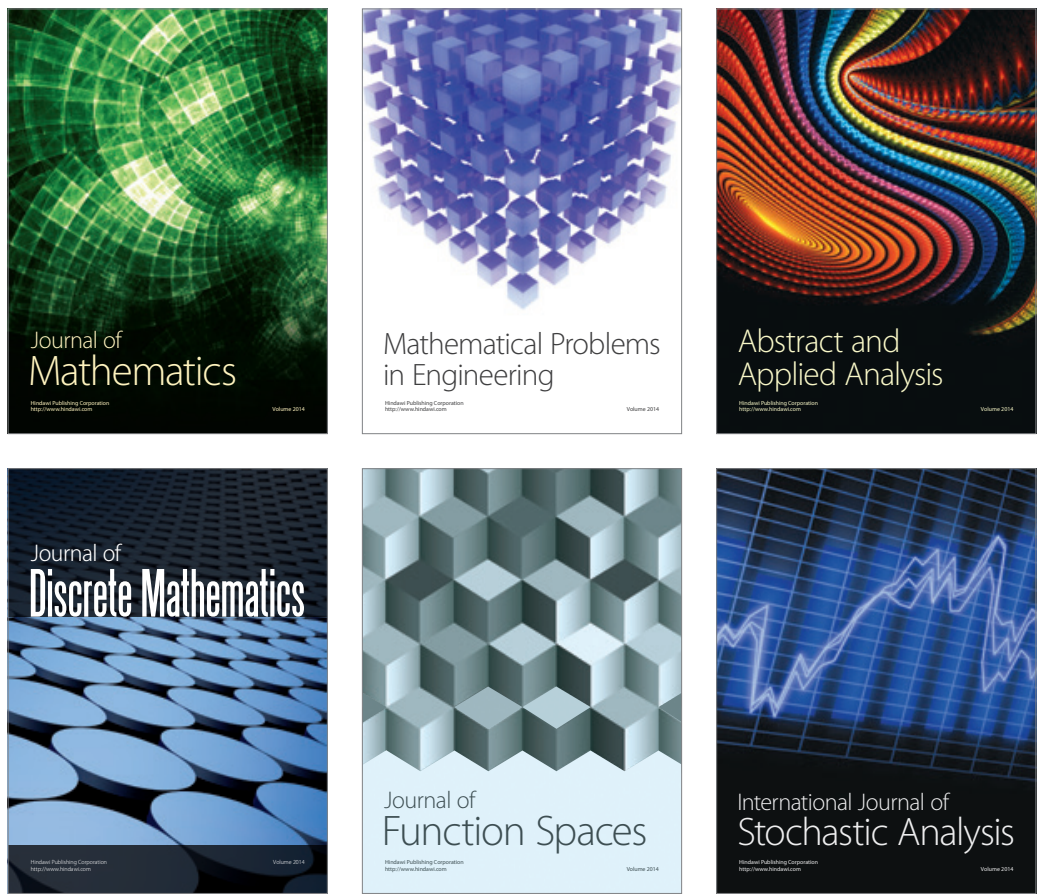

Journal of

Function Spaces

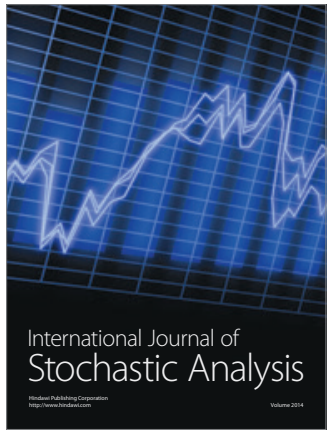

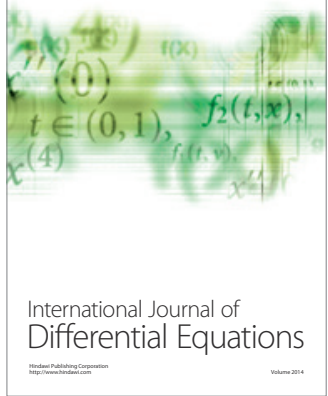
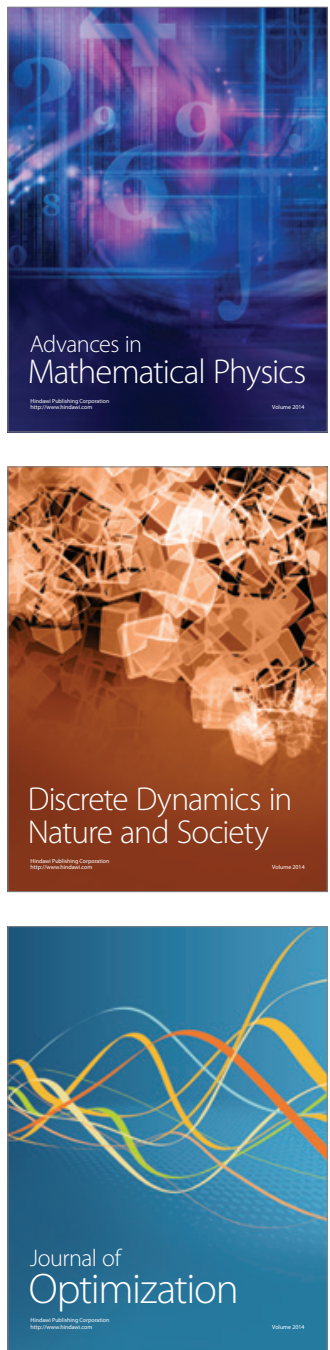